\title{
Single-cell biology meeting marks rebirth of an old science
}

\author{
Sten Linnarsson*
}

\section{Abstract \\ A report on the third Single Cell Analyses meeting, held at the Cold Spring Harbor Laboratory, New York, USA, March 6-9, 2013.}

\section{Introduction}

More than 100 researchers gathered at a wintery Cold Spring Harbor in March for the third Single Cell Analyses meeting. Where the first meeting in 2009 was tiny, and the second two years later was still quite small, at this year's meeting the attendance was strong, and there was a definite feeling that this young field is here to stay. The contrast with that first meeting, four years ago, was stark. Then, participants were often struggling to even obtain data from single cells, much less make sense of it. This year, the technical challenges had clearly been fought, if not yet completely mastered, and solid progress was reported on many fronts.

Some things had not changed, of course: the atmosphere at Cold Spring Harbor, always hospitable and efficient; the beautiful campus this time clad in a late but heavy winter snowfall; the friendliness and good judgment of the organizers, Nancy Allbritton (University of North Carolina, USA), Jim Eberwine (University of Pennsylvania, USA) and Scott Fraser (California Institute of Technology, USA); and the ample opportunities for informal chats and chance encounters in the dinner hall and evening bar.

In a sense, single-cell analysis is as old as cell theory itself, going back all the way to the invention of microscopy and the first observations of individual cells by Robert Hooke. But in another sense, we are currently witnessing the birth of a new cross-disciplinary science. This is because only very recently has it become possible through imaging and sequencing especially - to assay

*Correspondence: sten.linnarsson@ki.se

Laboratory of Molecular Neurobiology, Department of Medical Biochemistry and Biophysics, Karolinska Institutet, SE-171 77 Stockholm, Sweden large numbers of single cells for multiple cellular properties in a quantitative manner. The result has been a new appreciation for the diversity of cells, the heterogeneity of cellular responses, and the way that population measurements can sometimes conceal and even completely misrepresent the true complexity of life.

\section{Looking at single cells}

Some of the most impressive developments, as usual, were reported in imaging. Martin Chalfie (Columbia University, USA), the 2008 Nobel Laureate for his work on green fluorescent protein (GFP), opened the meeting with a look back at the development of GFP as a reporter, and how it is now used in his lab to dissect the development of touch sensory neurons in Caenorhabditis elegans.

Along with powerful reporter proteins derived from GFP, improvements in imaging resolution and frame rate are bringing us ever closer to three-dimensional movies of the secret lives of cells. Hari Shroff (NIBIB, NIH, USA) showed how structured illumination microscopy (SIM) traditionally a rather slow method but capable of surpassing the resolution limit of the light microscope - can be extended to multifocal SIM (MSIM), allowing acquisition rates 10- to 100-fold faster than standard SIM. The result? Awesome movies of organelles zipping around inside single cells.

Similarly, Scott Fraser reported on recent progress in three-dimensional time-lapse whole-organism imaging, which has already furthered our understanding of embryogenesis by showing exactly when each cell divides, and where it goes. The dance of cells that seem to jostle and tug at each other in the embryo is not only fascinating to watch, but is sure to generate more rigorous data on developmental processes. For example, when the temporal resolution of image acquisition exceeds the rate of cell division and migration, the complete lineage tree of the organism can be deduced by tracing the three-dimensional path of each nucleus.

\section{Genomes and transcriptomes of single cells}

The relentless pace of development of DNA sequencing technology has not only resulted in scaling up (of 
throughput), but also in a scaling down (of input DNA requirements), to the point where it is now feasible to sequence both genomes and transcriptomes of single cells. This is the area where progress since the first Single Cell Analyses meeting was the most noticeable.

Xuyu Cai (Harvard Medical School, USA) reported that over $90 \%$ of the genome can be consistently recovered from single neuronal nuclei, with surprisingly even coverage. She applied single-genome sequencing to look at patterns of somatic mutations in normal human brains, finding both L1 transposition events and copynumber and single-nucleotide variations occur in normal brain development.

Kun Zhang (University of California, San Diego, USA) showed a novel whole-genome amplification method, based on amplifying single cells in single microwells and then recovering the locally amplified product. The result was a significantly more even genome coverage, with a quality approaching that of bulk genome sequences.

Gene expression analysis in single cells has also made great strides. Several groups showed high quality data from single cells, both using single-mRNA molecule detection by imaging (for example, Alexander van Oudenaarden of the Hubrecht Institute, the Netherlands, and MIT, USA, and Sanjay Tyagi of the University of Medicine and Dentistry of New Jersey, USA) and using RNA-seq (for example, our own work and that of Gouping Fan of the University of California, Los Angeles, USA). There was a clear trend towards more accurate measurements as well as the analysis of larger numbers of cells. With more accurate measurements comes the ability to study subtle quantitative processes such as transcriptional noise; and with large cell numbers, the ability to study intercellular heterogeneity. Expect more of these trends in the future.

\section{Proteomes and microfluidics}

Two more trends were evident at the meeting. The first was the use of microfluidic devices tailored to the capture and analysis of single cells. This is a natural fit, of course, and there are now also the beginnings of a commercial market for single-cell analysis instruments outside of imaging (for example, the Fluidigm $\mathrm{C} 1$ AutoPrep for RNA-seq and quantitative PCR). Carl Hansen (Institute for Systems Biology, University of British Columbia, USA) showed how microfluidic devices could be used to analyze RNA and microRNA from very large numbers of single cells.

The second was single-cell proteomics, traditionally a very difficult proposition. In a very impressive talk, Sean Bendall (Stanford University, USA) showed how the trajectory of B cell development could be dissected in detail using mass cytometry, a relatively new method capable of simultaneous measurement of several tens of proteins in single cells with extremely high throughput.

\section{Conclusions}

The meeting was not characterized by a single major development, but rather by good solid progress on all fronts. It is clear by now that single-cell analysis is here to stay, and that it is poised to have a major impact on many disciplines. Conspicuous by its absence was any hint of clear applications in the clinic, probably because singlecell analysis remains technically challenging. I expect this to change rapidly in the coming few years as technology matures, and experimental designs become increasingly sophisticated. In particular, cancer would seem a natural first clinical application, because of the natural genomic, transcriptomic, and proteomic heterogeneity of tumors, and because multiple tiny subpopulations of cells (for example, cancer stem cells, circulating tumor cells and treatment-induced drug-resistant clones) play such an outsized role in the disease.

Competing interests

The author declares they have no competing interests.

Abbreviations

GFP, green fluorescent protein; MSIM, multifocal structured illumination microscopy; SIM, structured illumination microscopy.

Published: 19 April 2013

doi:10.1186/gb-2013-14-4-305

Cite this article as: Linnarsson S: Single-cell biology meeting marks rebirth of an old science. Genome Biology 2013, 14:305. 